\section{Insecticidal RNAs in green disguise}

The Colorado potato beetle $(\mathrm{CPB})$ is a notorious insect pest of not only potatoes but also tomatoes and eggplants. Both adults and larvae feed on leaves and can skeletonize entire plants, causing devastating effects on crop yields. Insecticides are currently the main method of bee-

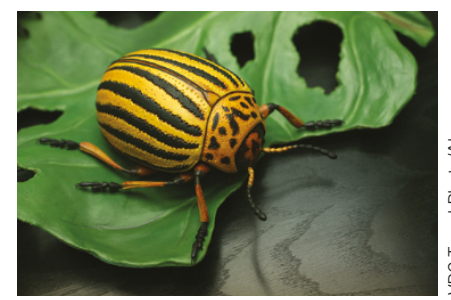

tle control, but the CPB is rapidly developing resistance. Transgenic plant technology offers an alternative strategy for crop protection, and one approach has been the expression of exogenous double-stranded (ds)RNAs that target essential insect genes to trigger a lethal RNAinterference (RNAi) response in feeding bugs. However, this tactic is hampered because insecticidal dsRNAs are unstable and are processed by the plants' own RNAi machinery into short interfering RNAs, which are less effective in causing a systemic RNAi response in pests. Bock and colleagues now report a new strategy that circumvents the problem. They stably expressed long dsRNA from the genome of chloroplasts, plant organelles evolutionary derived from cyanobacteria, which lack the RNAi pathway. In this manner, dsRNAs accumulate to high levels, without causing a visible phenotype in the potato plants in regard to growth and tuber production. But are these plants protected against the hungry mouths of the potato beetles? Indeed, Bock and colleagues showed that chloroplast-transformed plants producing dsRNA targeting the $\mathrm{CPB} \beta$-actin gene were lethal to $\mathrm{CPB}$ larvae and were protected against herbivory, even from adult beetles. Plants that expressed the same dsRNAs from the nuclear genome were less well shielded and caused no larval mortality, although larval growth was still retarded. The authors confirmed that the toxic effects were due to repression of the target gene, with obvious consequences on the CPB actin cytoskeleton, especially in cells of the midgut. The potential for RNAi technology in the control of insect pests is great, and shifting dsRNA synthesis from the plant nucleus to the chloroplast is a major step forward for optimization of dsRNA delivery from plants to insects. (Science 347, 991-994, 2015)

AS

\section{Helping dengue along its way}

Dengue, a mosquito-borne viral disease, is a major public health concern in tropical regions. Clinical manifestations range from flu-like symptoms to the more severe dengue hemorrhagic fever (DHF), and there are currently no vaccines or specific medications to treat the infection. The disease is caused by a group of related, but antigenically distinct, enveloped single-stranded RNA viruses (DENV). In infected cells, viral particle assembly takes place in the endoplasmic reticulum (ER). The two structural glycoproteins E and prM are integrated into the lipid envelope of the nascent virion in the ER lumen, and the particles are then trafficked through the cis- and trans-Golgi network. There, the 'pr' propeptide of prM is cleaved to yield mature DENV particles, which are then released. However, little is currently known about host factors involved in DENV egress. Production of recombinant subviral particles (RSPs) upon ectopic expression of prM and $\mathrm{E}$ in cells provides a safe, convenient model system to study virus secretion. This system was previously used to demonstrate that depletion of Arf4 and Arf5, two small GTPases involved in the formation of trafficking vesicles, results in entrapment of RSPs in the ER. Interestingly, depletion of Arf4 and Arf5 also inhibits the retrograde cycling of KDEL receptors (KDELRs), proteins involved in the retention of resident soluble proteins in the ER lumen. Therefore, Bruzzone, Wang and colleagues investigated the involvement of KDELRs in DENV trafficking and found that siRNAmediated knockdown of these receptors reduced release of RSPs and DENV from cells. Moreover, KDELRs colocalized with prME in DENVinfected or prME-expressing cells, thus suggesting a possible physical interaction. Biochemical analyses indeed showed that prM interacts with KDELRs through its N-terminal pr peptide in the ER. Mutagenesis identified amino acid residues in prM that mediate KDELR binding in vitro. Functionally, mutation of these residues did not inhibit RSP assembly in the ER but did reduce particle trafficking to the cis-Golgi. The authors thus define a new intracellular receptor required for DENV egress and show that host factors play a part in sorting viral particles during their exit from cells. (Cell Rep. 10, 1496-1507, 2015) SG

\section{Keeping autophagy at bay}

Nutrient deprivation induces a number of physiological responses, including autophagy. This evolutionarily conserved process allows breakdown and recycling of cellular components and is regulated by the ATG genes in yeast and their orthologs in higher organisms. However, the transcriptional control of these genes is still incompletely understood. In a screen to identify factors regulating ATG gene expression upon nitrogen starvation, Klionsky and colleagues have now identified Rph1 as a transcriptional repressor of a subset of ATG genes under nutrient-replete conditions. Rph1 is a histone demethylase that acts to reverse methylation of Lys36 of histone H3. However, the demethylase activity of Rph1 is dispensable for the autophagic response, although promoters of Rph1-responsive ATG genes contain Rph1-binding sites. Zinc-finger motifs in Rph1 were found to interact with these sites in the ATG7 promoter and were required for Rph1's ability to inhibit autophagy, thus suggesting that DNA binding mediates Rph1's function as a transcriptional repressor. Nitrogen starvation led to decreased levels of Rph1 protein and induced its phosphorylation. Rim15, a kinase that integrates signals from various nutrient-sensing pathways, was shown to mediate Rph1 phosphorylation in this context. Knockdown experiments in HeLa cells demonstrated that KDM4A, a mammalian Rph1 homolog, was similarly involved in repressing transcription of certain Atg genes. KDM4A knockdown also led to an increase in several autophagic markers. In addition, autophagy induced by the mTOR inhibitor Torin 1 resulted in phosphorylation of KDM4A and reduced its cellular levels. Together, these results suggest that Rph1's function is evolutionarily conserved. Although Rph 1 had previously been implicated in nutrient signaling, the current study defines its role in repressing autophagy-related genes under nutrient-rich conditions and provides insights into the signal-transduction pathway linking nutrient sensing and Rph1mediated regulation of autophagy. (Curr. Biol. 25, 546-555, 2015) 